\title{
RMS Value Measurement Using a Meter Based on ARM Cortex-MO Microprocessor
}

\author{
Ajakida Eski, Orion Zavalani, and Denald Komici
}

\begin{abstract}
In an electrical grid at the transmission and distribution system level, to guarantee the system security and the power quality, the RMS value of voltage and current is an important issue. We calculate the RMS value by processing the samples of a signal in the time domain. A software based explicit RMS converter is presented to transform the alternating voltage and current waveforms into their RMS values. This paper briefly covers the mathematics of RMS and offers an algorithm of RMS measurements based in FIR digital filter, which are more versatile when compared to the analogue filters in their characteristics such as programming flexibility, ability to handle both low as well as high frequency signals accurately. The measurement system is designed using ARM Cortex MO, PSoC4, CY8C4245AXI-483 architecture which is responsible for executing algorithms required for the calculation and ensures high performances and low cost. To check the accuracy of the metering system, the proposed metering values are compared to the readings of a standard FLUKE metering and it is concluded that the meter has been successfully completed. Since the proposed approach provides low cost solution for true RMS metering, it can be used in industrial and commercial applications especially as Digital panel meters and harmonic detectors.
\end{abstract}

Index Terms - Algorithm; Digital Filter; RMS Value.

\section{INTRODUCTION}

In an electrical grid at the transmission system level, to guarantee the system security, voltage RMS control is an important issue. Whereas at the distribution system levels, this is an issue about power quality. Variations in voltage magnitude can affect the performance of electricalelectronic end-user equipment. Therefore, the Root Mean Square (RMS) is a fundamental measurement of the magnitude of an alternating signal and variations in the voltage RMS is limited to a range by international standards.

The power electronics and switching devices have nonlinear voltage-current characteristics. Using these devices are introduced the harmonic currents in the power system which change the sinusoidal nature of the ac power current and voltage and increase $I^{2} R$ losses in the system [1]. Since the analog instruments used for RMS measurement are calibrated for sinusoidal signals they introduce appreciable error when total harmonic current distortion is more than $100 \%$. For accurate measurements of RMS values need to replace them with modern digital meters.

The AC measuring methods are classified as

a) Rectify and average

Publishedn on November 15, 2017

A. Eski, O. Zavalani and D. Komici are with the Faculty of Electrical Engineering, Polytechnic University of Tirana, Albania. (e-mail : ajakidaeski@yahoo.com) b) Thermal

c) Analog computation (Direct and Indirect computation)

d) Sampling technique based computation

The Rectify-and-average method is accurate only when the input waveform is an undistorted sine wave otherwise the serious errors develop. Thermal method is a high bandwidth solution having high design complexity and cost constraints, while Analog computation method has the problems of bandwidth restrictions [2]. The Sampling technique based measurement, calculate the RMS value using digital signal processing based on hardware or software.

In this paper, is presented the software based explicit RMS converter to transform the alternating voltage and current waveforms into RMS values. The measurement system is designed using ARM Cortex MO microcontroller which processes the sampled data to calculate the true RMS value. The proposed approach ensures high performances and low cost. Since the proposed approach provides low cost solution for true RMS metering, it can be used in industrial and commercial applications especially as Digital panel meters and harmonic detectors.

\section{DESCRIPTION OF ALGORITHM FOR MEASURING OF THE RMS VALUES}

The Root Mean Square (RMS) is a fundamental measurement of the magnitude of an alternating signal. Its definition can be both practical and mathematical. In mathematics, the standard deviation [3] is a statistical measure used to quantify the signal deviation from the mean value, which means that the deviation measures only the AC component of the signal. Another term commonly used in electronics is RMS value (root-mean-square) which measures both the AC and DC components of the signal. When the RMS value of a signal is identical to its standard deviation then the signal has no DC component.

Defined practically in electrical engineering, the RMS value or effective value [4] of a given AC signal is the amount of DC required to produce an equivalent amount of heat in the same resistive load.

Digital signal processing is performed in the time or frequency domain. In this paper, although the time domain method calculates the RMS value of the whole signal spectrum and not of the harmonic components, we use it because it is simpler and more accurate [5] than the frequency domain methods.

The mathematical definition of RMS value of a signal is

$\mathrm{X}_{\mathrm{RMS}}=\sqrt{\frac{1}{\mathrm{~T}} \int_{0}^{\mathrm{T}}\left[\mathrm{x}(\mathrm{t})^{2}\right] \mathrm{dt}}$ 
where $X_{R M S}$ is the RMS value, $x(t)$ is the instantaneous voltage of a function time, $T$ is the time measurement (in the classical approach, an integer multiple of the signal period). Squaring both sides of this equation yields

$X_{R M S}^{2}=\frac{1}{T} \int_{0}^{T}\left[x(t)^{2}\right] d t$

The integral can be approximated as a running average:

$$
\operatorname{Avg}\left[x(t)^{2}\right]=\frac{1}{T} \int_{0}^{T}\left[x(t)^{2}\right] d t
$$

Then (2) simplifies to

$X_{R M S}^{2}=\operatorname{Avg}\left[x(t)^{2}\right]$

Note that taking the square root of both sides of (4) yields

$X_{R M S}=\sqrt{\operatorname{Avg}\left[x(t)^{2}\right]}$

which is an alternate way of expressing the RMS value of the function.

This alternative way of expression of the RMS value overcomes the inherent limitation of direct calculation (1), which requires the precisely definition of the integration limits. This proposed method is known as an average power converter similar to the explicit RMS converter which has been used primarily for monolithic RMS/DC converters [6],[7].

RMS estimation for digitized signal is based on the following equation.

$X_{R M S} \cong \sqrt{\frac{1}{M \cdot N} \sum_{k=1}^{M \cdot N} x_{k}^{2}}$

where $x_{k}$ is the value of each signal sample, $M$ is the total number of cycles in the measurement period and $N$ is the number of samples/cycle. The voltage and current in an electrical power system are parameters that change slowly and they can be considered as constant values during the sampling period [8], [9]. Therefore, the measurement accuracy is not affected by averaging of the sample values during the measurement period.

In this paper, a software based explicit RMS converter is presented to transform the alternating voltage and current waveforms into their RMS values. This method requires the calculation of the square, average and square root functions for each new sample of the signal being analyzed. Fig. 1 shows the average RMS converter implementation.

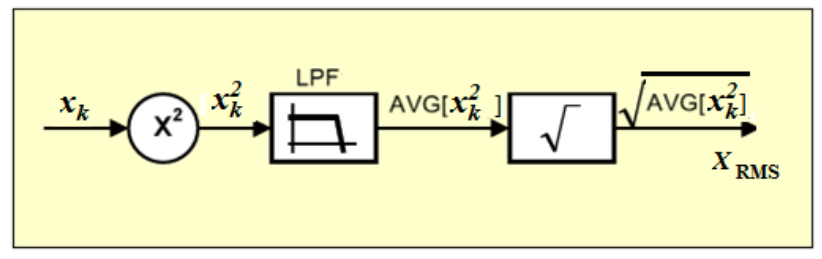

Fig.1. Explicit RMS current converter

This algorithm uses the moving average filter, which is a low-pass digital FIR for calculating the averaging of the samples over $\mathrm{M}$ number of cycles. FIR filters are digital filters with finite impulse response. They are also known as non-recursive digital filters as they do not have the feedback (a recursive part of a filter). The transfer function of the FIR filter approaches the ideal as the filter order increases. FIR filters can have an exactly linear phase response. The implication of this is that no phase distortion is introduced in to the signal by the filter. FIR filters are simple to design and they are extremely stable with respect both to time and temperature. The low sensitivity to filter coefficient quantization errors is an important property of FIR filters [10] that is valuable for their implementation on DSP processors or on an integrated circuit.

As the name implies, the moving average filter operates by averaging a number of points from the input signal to produce each point in the output signal. Using a recursive algorithm, in which the result of one calculation is used in future calculations, the moving average filter will run like lightning in computer. In fact, it is the fastest digital filter available.

\section{HARDWARE CONSIDERATIONS}

The proposed measurement system is based on a single low cost microcontroller for sampling and digital processing of the input signal. The ARM Cortex MO, PSoC4, CY8C4245AXI-483 microcontroller is responsible for executing algorithms required for calculation of RMS value.

The A/D Converter of microcontroller performs the digital conversion of all the input signals. The bandwidth and accuracy of measurement system are the two main specifications. The bandwidth of the system is decided by the sampling frequency while accuracy depends on resolution of the A/D Converter. The ADC's sample rate should be selected to be sufficient to meet the Nyquist rate for the type of measurement that will make (i.e., slightly greater than twice the highest frequency that is desired to be measured), otherwise, the high-frequency content will alias at a frequency inside the spectrum of interest (pass band). For measurements with a certain accuracy over a given range, the resolution (effective number of bits) of the $\mathrm{A} / \mathrm{D}$ Converter is calculated.

For achieving the accuracy of the order $1 \%, \mathrm{~A} / \mathrm{D}$ converter with 12 bit resolution and sampling rate of 3200 sample/second is sufficient. In this study, calculation of voltage RMS values are done at each period of $1 \mathrm{sec}$. ARM microcontroller of system communicates with the next level equipment as a monitoring PC via the Serial interface.

\section{TESTING, RESUlts AND DiscUSSION}

The performance of the measurement system has been evaluated in the laboratory for voltage measurement. Fig. 2 shows the implementation of laboratory testing.

To evaluate the performance, the proposed metering system has been tested in the Laboratory. We have performed the measurements both with the proposed system as well as with standard FLUKE meter. 


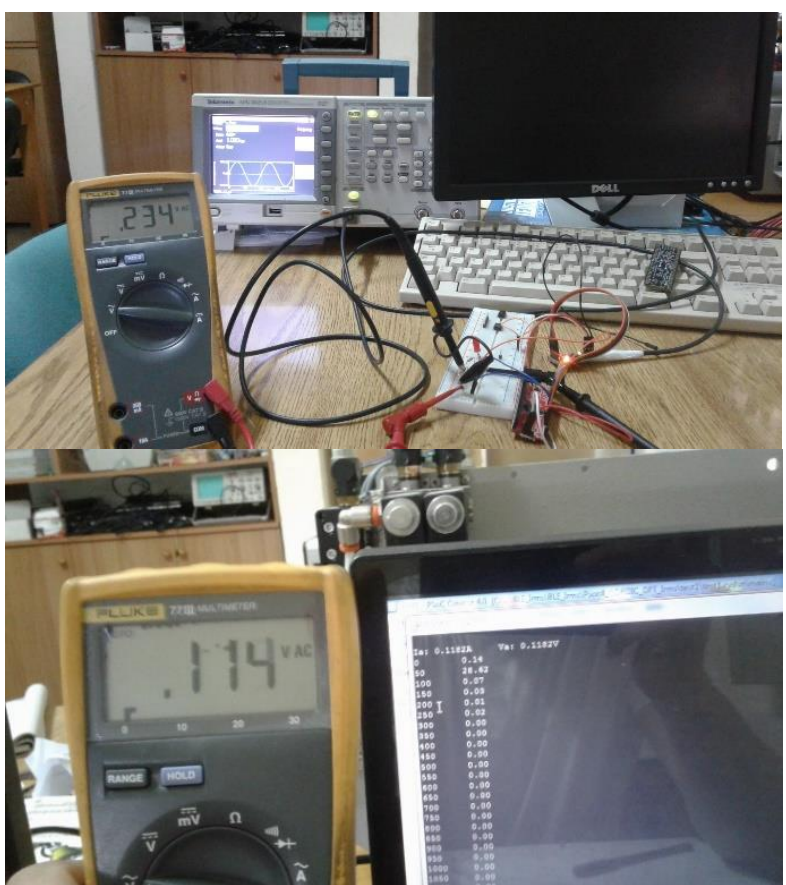

Fig.2. Photo of realized measurements

TABLE I: RESULTS OF MEASUREMENTS IN THE LABORATORY

\begin{tabular}{|c|c|c|c|}
\hline \multicolumn{3}{|c|}{ Measured RMS value of voltage (volt) } & \multirow{2}{*}{$\begin{array}{c}\text { Relative } \\
\text { error } \\
(\%)\end{array}$} \\
\hline $\begin{array}{c}\text { Function } \\
\text { generator } \\
\text { AFG3021B }\end{array}$ & $\begin{array}{l}\text { FLUKE 77III ( } \\
\text { standard meter) }\end{array}$ & $\begin{array}{c}\text { Proposed } \\
\text { measurement } \\
\text { system }\end{array}$ & \\
\hline 0,1 & 0,021 & 0,0218 & $-0,08 \%$ \\
\hline 0,2 & 0,045 & 0,0459 & $-0,09 \%$ \\
\hline 0,3 & 0,069 & 0,0697 & $-0,07 \%$ \\
\hline 0,4 & 0,094 & 0,0939 & $0,01 \%$ \\
\hline 0,5 & 0,117 & 0,1173 & $-0,03 \%$ \\
\hline ,6 & 0,141 & 0,1418 & $-0,08 \%$ \\
\hline 0,7 & 0,166 & 0,1662 & $-0,02 \%$ \\
\hline 0,8 & 0,193 & 0,1933 & $-0,03 \%$ \\
\hline 0,9 & 0,216 & 0,2165 & $-0,05 \%$ \\
\hline 1 & 0,237 & 0,2372 & $-0,02 \%$ \\
\hline
\end{tabular}

The FLUKE meter readings are considered as true values. From the measured values has been calculated the relative error of measurement.
Table 1 shows the measured RMS values of the voltage with the standard meter and the proposed system as well as the calculated values of the relative measurement error.

\section{CONCLUSION}

In this paper, we have presented the RMS value measurement executing a digital filter based algorithm in an ARM Cortex-MO microcontroller. We have selected an A/D converter with 12 bit resolution and sampling rate of 3200 sample/second so that the measurement system meets the specified bandwidth and accuracy. The calculated error values presented in Table 1 meet the required measurement accuracy by demonstrating that the proposed measurement system has been successfully developed. Since the proposed approach provides low cost solution for true RMS measurement, it can be used in industrial and commercial applications.

\section{REFERENCES}

[1] IEEE 519-1992: "Recommended practices and requirements for harmonic control in electrical power system",IEEE1992

[2] Gerard N. Stenbakken, "A wideband Sampling Wattmeter," IEEE Transaction on Power Apparatus and Systems, Vol. PAS 103, No.10 October 1984

[3] Steven W. Smith, Digital Signal Processing, Second Edition, 1999

[4] Charles K. Alexander, Matthew N.O. Sadiku, Fundamentals of Electric Circuits

[5] Martin Novotny, Milos Sedlacek, RMS value measurement based on classical and modified digital signal processing algorithms, Measurement 41 (2008) 236-250

[6] RMS to DC Conversion Application Guide, Charles Kitchin, Lew Counts (2nd Edition, analog Devices, 1986, USA)

[7] Linear Circuit Design Handbook, Hank Zumbahlen (editor) (Elsevier-Newnes, 1st Edition, 2008, USA)

[8] P.Petrovic, "New Digital Multimeter for Accurate Measurement of Synchronously Sampled AC Signals," IEEE Trans. Instrum.Meas., vol. 53, pp. 716-725, June 2004.

[9] Gerard N. Stenbakken, “A wideband Sampling Wattmeter," IEEE Transaction on Power Apparatus and Systems, Vol.PAS 103,No.10 October 1984

[10] Taylor, Fred J., Digital filters: principles and applications with MATLAB. IEEE series on digital \& mobile communication; 30 ISBN 978-0-470-77039-9,2012 